\title{
Systemic Lupus Erythematosus and Irritable Bowel Syndrome: Is Blastocystis Hominis the Missing Piece of the Puzzle?
}

\author{
Mohammed M. Keshawy ${ }^{*}$, and Maha M. Alabbassy
}

Departments of ${ }^{1}$ Internal Medicine, and ${ }^{2}$ Parasitology, Faculty of Medicine, Suez Canal University, Egypt

\begin{abstract}
Background: Irritable bowel syndrome (IBS) is a chronic functional gastrointestinal disease presenting clinically by abdominal pain with alteration of bowel habits. Although IBS has uncertain etiology, chronic gut inflammation due to persistent exposure to an infectious agent including Blastocystis sp. was proposed. Aim: to determine the prevalence of Blastocystis hominis infestation in Systemic lupus erythematosus (SLE) patients and determining the immunomodulatory effect of Blastocystis hominis on SLE pathogenesis. Subjects and Methods: A total number of 84 patients attending the SCU hospital outpatient clinics (40 IBS patients / 24 SLE patients / 20 healthy controls) were enrolled in the study and a stool and blood samples were collected. Blastocystis was detected by PCR and serum IL- 6 assay by ELISA. Results: Among IBS patients, Blastocystis sp. could be detected at a frequency of $2.5 \%(1 / 40), 27.5 \%(11 / 40)$ using direct microscopy and PCR assay respectively while among SLE/IBS patients, it could be detected at a frequency of $8.33 \%(2 / 24)$, $41.66 \%$ (10/24). IL-6 assay was higher in PCR positive patients in all study groups. Conclusions: The prevalence of Blastocystis hominis infestation is higher in SLE patients who had IBS compared to IBS patients or asymptomatic controls; with evidence of IL- 6 increase in their sera suggesting an immunomodulatory interaction between SLE and Blastocystis hominis.
\end{abstract}

Keywords: IBS, SLE, Blastocystis hominus

\section{Introduction}

Irritable bowel syndrome (IBS) is considered the commonest cause of referral to gastroenterologists worldwide creating an economic burden for healthcare providers. Several studies have examined the prevalence of IBS in different geographic regions, and in general, have found the prevalence of IBS to be higher in industrialized nations $^{(1,2)}$. The actual etiology of IBS is still questionable, and many factors interplay such as psychosocial factors, GIT dysmotility and GIT hypersensitivity(3). Several parasites including E. histolytica, Giardia sp., B. hominis, and Trichinella sp. have been discussed as contributing factors to the development of IBS, though the relationship is less well defined ${ }^{(4)}$. Blastocystis hominis is a common gut parasite which is transmitted through the fecal-oral route and exists in many morphological forms such as vacuolar, granular, amoeboid, cyst, avacuolar and multi-vacuolar forms ${ }^{(5)}$. At least 17 subtypes (STs) of Blastocystis have been identified, with 9 of them (subtype 1

*Corresponding Author: mmkeshawy@yahoo.com 
[ST-1] to ST-9) detected in humans ${ }^{(6)}$. In 2012; Chandramathi et al, reported that Blastocystis in immunosuppressed patients due to chemotherapy tend to exacerbate IBS symptoms( $(7)$, and the same was reported in HIV patients. Some in vitro studies showed the cytopathic effects of Blastocystis sp. on mammalian cell cultures like the study of Long et al, in 2001,which showed that $24 \mathrm{~h}$ incubation with Blastocystis sp. ST1 cells or culture filtrates induced the production of interleukin IL-8 and granulocyte-macrophage colony-stimulating factor, suggesting that the parasite was able to modulate the host immune response ${ }^{(8)}$ then the later study done Puthia et al, in 2008 showed that IL-8 and IL-6 production from human colonic epithelial cells (HT84) was induced by cysteine proteases from Blastocystis spp. ST4 in a nuclear factor $k B$ dependent manner ${ }^{(9)}$. Blastocystis exhibits a subtype-dependent upregulation of proinflammatory cytokines and mitogen-activated protein kinase (MAPK) activation in macrophages as Blastocystis ST4 (WR-1) and ST-7 (B) induces the expression of IL- 6 , IL-1 $\beta$, and TNF- $\alpha$, supporting serine proteases as virulence factors of Blastocystis(10). Systemic lupus erythematosus (SLE) is an autoimmune disease of unknown pathogenesis which can cause $\mathrm{Gl}$ symptoms due to primary gastrointestinal disorders or complications of therapy or SLE itself ${ }^{(11)}$. Patients who have active SLE show increased IL- 6 levels in their sera which is correlated with disease activity or anti-DNA levels as showed in many other studies $^{(12)}$. Elevated IL-6 levels are associated with increased B-cell activity and production of autoantibodies while the secretion of anti-DNA antibodies were reduced by neutralizing IL- 6 and restored by adding exogenous IL- 6 in vitro ${ }^{(13)}$. In addition to its systemic actions; IL- 6 is a key player in the local inflammatory response encountered in renal, cardiac, pulmonary and neuropsychiatric manifestations of SLE with a growing evidence of its involvement in joint damage in such patients ${ }^{(14)}$. The rationale of the present study relies on the fact that cytokines are responsible of intercellular signaling which orchestrate the interaction of immune cells during immune responses and sharing IL- 6 as a key player between immune disorders like IBD , urticaria , Hashimoto's thyroiditis and the possible relationship demonstrated by many studies to Blastocystis hominis may points to a link which worth a closer look specially in SLE with disease natural history of multiorgan affection ; this serves as an interesting area to investigate this point.

\section{Patients and Methods}

The study aims at determining the prevalence of Blastocystis hominis infestation in patients who have IBS and SLE patients who have IBS compared to normal healthy controls and determining the immunemodulatory effect of Blastocystis hominis on SLE pathogenesis. Using a convince sample technique over a one month period; a total number of 84 subjects (40 IBS patients/24 SLE with IBS patients/20 healthy controls) were enrolled in the study who are attending the internal medicine outpatient clinics (gastroenterology and specialized SLE care services) in Suez Canal University hospitals, Ismailia. SLE patients meeting the Systemic Lupus Collaborating Clinics (SLICC) criteria for SLE diagnosis(15) were included in the study as follows: adult patients $\geq 18$ years, on long term immunosuppressive therapy $>12$ month and had a stable course documented by normal $\mathrm{C}_{3}$ and $\mathrm{C}_{4}$ at least twice in their records during the previous 6 months while diabetic patients were excluded from all study groups to avoid confounding with diabetic gastroparesis. Stool and blood samples were collected. Blastocystis was detected by PCR examining 3 stool samples at different times due to the 
parasite's irregular shedding in stool. The stool samples were collected in labeled plastic vials without preservatives and subjected to macroscopic examination, direct microscopy with saline/iodine and PCR. Genomic DNA of $B$. hominis was extracted by using DNAzol reagent (Gibco BRL/Life Technologies, Inc., Grand Island, N.Y.) according the manufacturer's instructions. PCR technique was performed as previously described by Yoshikawa et al, 2000(16) and Blastocystis sp. subtypes were identified using classification proposed by Stensvold et al, in 2007, in which Blastocystis sp. subtypes are identified as $n$, where $n$ is a designation developed through phylogenetic classification by small-subunit ribosomal DNA analysis (Table 1) ${ }^{(17)}$.

Table 1. Blastocystis hominis gene sequencing and diagnostic primer sets according to Blastocystis sp. subtypes

\begin{tabular}{|c|c|c|c|c|}
\hline $\begin{array}{c}\text { B. hominis } \\
\text { subtype }\end{array}$ & Primer & Forward sequence & Reverse sequencing & Size (bp) \\
\hline 1 & SB83 & GAAGGACTCTCTGACGATGA & GTCCAAATGAAAGGCAGC & 351 \\
\hline 2 & SB155 & ATCAGCCTACAATCTCCTC & ATCGCCACTTCTCCAAT & 650 \\
\hline 3 & SB227 & TAGGATTTGGTGTTTGGAGA & TTAGAAGTGAAGGAGATGGAAG & 526 \\
\hline 4 & SB332 & GCATCCAGACTACTATCAACATT & CCATTTTCAGACAACCACTTA & 338 \\
\hline 5 & SB336 & GTGGGTAGAGGAAGGAAAACA & AGAACAAGTCGATGAAGTGAGAT & 317 \\
\hline 6 & SB332 & GCATCCAGACTACTATCAACATT & CCATTTTCAGACAACCACTTA & 338 \\
\hline 7 & SB337 & GTCTTTCCCTGTCTATTCTTGCA & AATTCGGTCTGCTTCTTCTG & 487 \\
\hline
\end{tabular}

Blood samples were collected in EDTA tubes and centrifuged to obtain the serum samples which were kept at $-20^{\circ} \mathrm{C}$ until serum IL- 6 was measured using EnzymeLinked Immunosorbent Assay (Bio-Rad ELISA kit). IBS was diagnosed based on ROME IV diagnostic criteria for functional gastrointestinal disorders as follows: IBS with predominant constipation (IBS-C), IBS with predominant diarrhea (IBS-D), IBS with mixed bowel habits (IBS-M) and IBS unclassified (IBS-U) ${ }^{(18)}$. Statistical analyses was done using IBM SPSS version 24 were Chi-square test was used to examine the differences in prevalence of Blastocystis in the study groups. IL- 6 measurement was analyzed using Student $T$ test. Statistical significance was defined as a $p$ value of $<0.05$.

\section{Results}

Table 2. summarizes the sociodemographic data of the participated patients and controls demonstrating clearly the female gender predominance in SLE/IBS were $100 \%(n=24)$ of the studied population were females which is expected by the natural history of SLE while in IBS group the female forms $57.5 \%(n=23)$ and the studied population mean ages were relatively the same and most of them were from urban rather rural areas. The SLE patients enrolled in the study has a mean disease duration of 7.02 years and $5 / 24$ has hypertension as a comorbid condition and they were maintained in various treatment protocols most commonly Steroid / AZA (11/24) and Steroid/ MMF (7/24) as demonstrated in table 3. Blastocystis sp. was detected in IBS group with direct microscopy in $2.5 \%(1 / 40)$ of patients while using PCR assay the detection improved to $27.5 \%$ (11/40). The same was in SLE/IBS patients, it was detected with direct microscopy in 8.33\% (2/24) and with PCR assay in $41.6 \%$ (10/24) of the studied patients. Direct wet mount examination of control group failed to detect Blastocystis sp. while PCR assay was able to detect 4 carriers (20\%) (Table 4). Blastocystis sp. subtype identification 
showed that ST 3 was the most common subtype as in IBS group, it represented $17.5 \%$ (7/40), in SLE/IBS group, it represented $25 \%(6 / 24)$ and finally in control group, it represented $50 \%$ of (2/4) encountered in carriers. $41.66 \%(10 / 24)$ of the studied SLE patients have Blastocystis hominis infestation mainly subtypes 2 and 3 (4 and 6 patients respectively) which is much higher when compared to patients with IBS or healthy controls $(27.5 \%$ and $20 \%$ respectively) [Table 5 and figure 1].

Table 2. Sociodemographic characteristic of studied patients and control groups

\begin{tabular}{|l|c|c|c|c|c|c|}
\hline & \multicolumn{2}{|c|}{ IBS group $(n=40)$} & \multicolumn{2}{c|}{ SLE/IBS $(n=24)$} & \multicolumn{2}{c|}{ Control $(n=20)$} \\
\hline Age (mean \pm SD) & \multicolumn{2}{|c|}{$29.25 \pm 6.73$} & \multicolumn{2}{c|}{$27.78 \pm 6.43$} & \multicolumn{2}{c|}{$27.47 \pm 6.68$} \\
\hline Gender & $\mathrm{N}$ & $\%$ & $\mathrm{n}$ & $\%$ & $\mathrm{n}$ & $\%$ \\
$\bullet \quad$ Male & 17 & 42.5 & -- & - & 12 & 60 \\
- Female & 23 & 57.5 & 24 & 100 & 8 & 40 \\
\hline Residence & & & & & & \\
- Urban & 28 & 70 & 19 & 79.16 & 13 & 65 \\
- Rural & 12 & 30 & 5 & 20.84 & 7 & 35 \\
\hline
\end{tabular}

Table 3. Clinical and treatment characteristics of SLE patients

\begin{tabular}{|l|c|}
\hline & $\operatorname{SLE}(\mathrm{N}=24)$ \\
\hline Disease duration & $7.02 \pm 3.94$ \\
\hline Type of immune suppression & 3 \\
- Steroid & 11 \\
- Steroid / AZA & 7 \\
- Steroid / MMF $\quad$ Steroid / CYC & 3 \\
\hline Comorbid & 5 \\
\hline - Hypertension & \\
AZA = azathioprine; MMF = mycofenolate moftil, CYC= cyclophosamide
\end{tabular}

Table 6 provides a detailed information on the symptomatic status of the patients from whom Blastocystis was isolated using PCR based on ROME-IV classification for IBS were the total symptomatic patients were only $32.8 \%$ (21/64) while the major set were asymptomatic and the most common presentation was constipation in $17.18 \%$ (11/64) of total symptomatic patients. Moreover; IL- 6 was higher in PCR positive patients in all study groups being the highest in SLE patients and the least in control group (29.2 \pm 2.9 vs. $13.8 \pm 1.8 \mathrm{pg} / \mathrm{dl})$ and was statistically significant compared to PCR negative samples (table 7).

\section{Discussion}

Blastocystis sp. was established as a probable causal agent in patients suffering from $\mathrm{IBS}^{(19)}$, however, the exact pathogenic mechanisms is not clearly delineated, except some protease enzymes of Blastocystis sp. that cause mucosal disruption and dysbiosis ${ }^{(20)}$. Most of the studies related to association of Blastocystis sp. in IBS cohorts were conducted in MiddleEast, Southeast Asia and South-America. In the present study, among IBS patients, Blastocystis sp. was detected at a frequency of $2.5 \%$ (1/40), 27.5\% (11/40) using direct microscopy and $P C R$ assay respectively, while among SLE/IBS patients, it could be detected at a frequency of $8.33 \%$ (2/24), 41.66\% (10/24). Direct wet mount examination of control group reveals no detectable samples while by using PCR, only 4 subjects were positive (20\%). Concerning 
the importance of detection of the Blastocystis sp. and PCR being an objective method has been advocated as the method of choice for the detection ${ }^{(21)}$. Studies from Turkey(22) Pakistan ${ }^{(23)}$ and Italy ${ }^{(24)}$ have also reported significant association of Blastocystis sp. in IBS patients compared to healthy controls. Nevertheless, contrasting results from Mexican studies ${ }^{(25)}$ have shown no difference in the frequency of Blastocystis sp isolation between IBS patients and the controls.

Table 4. Blastocystis identification method in stool samples

\begin{tabular}{|l|c|c|c|c|c|c|}
\hline \multirow{2}{*}{} & \multicolumn{2}{|c|}{ IBS group $(\mathrm{n}=40)$} & \multicolumn{2}{c|}{ SLE/IBS $(\mathrm{n}=24)$} & \multicolumn{2}{c|}{ Control $(\mathrm{n}=20)$} \\
\cline { 2 - 7 } & $\mathrm{n}$ & $\%$ & $\mathrm{n}$ & $\%$ & $\mathrm{n}$ & $\%$ \\
\hline Direct microscopy & 1 & 2.5 & 2 & 8.33 & - & - \\
PCR & 11 & 27.50 & 10 & 41.66 & 4 & 20 \\
\hline
\end{tabular}

Table 5. Blastocystis genotypes identified by PCR in each group

\begin{tabular}{|l|c|c|c|c|c|c|c|c|}
\hline & \multicolumn{2}{|c|}{ Subtype 1 } & \multicolumn{2}{c|}{ Subtype 2 } & \multicolumn{2}{c|}{ Subtype 3 } & \multicolumn{2}{c|}{ Total } \\
\cline { 2 - 9 } & $\mathrm{n}$ & $\%$ & $\mathrm{n}$ & $\%$ & $\mathrm{n}$ & $\%$ & $\mathrm{n}$ & $\%$ \\
\hline IBS $(\mathrm{n}=40)$ & 1 & 2.50 & 3 & 7.50 & 7 & 17.50 & 11 & 27.50 \\
\hline SLE/IBS $(\mathrm{n}=24)$ & - & - & 4 & 16.66 & 6 & 25 & 10 & 41.66 \\
\hline Control $(\mathrm{n}=20)$ & - & - & 2 & 50 & 2 & 50 & 4 & 20 \\
\hline
\end{tabular}

Table 6. Distribution of PCR positive Blastocystis hominis infestation according to ROME-IV IBS subclass

\begin{tabular}{|l|c|c|c|c|c|c|}
\hline \multirow{2}{*}{} & \multicolumn{2}{|c|}{ IBS group $(\mathrm{n}=40)$} & \multicolumn{2}{c|}{ SLE/IBS $(\mathrm{n}=24)$} & \multicolumn{2}{c|}{ Total symptomatic patients $(\mathrm{n}=64)$} \\
\cline { 2 - 7 } & $\mathrm{n}$ & $\%$ & $\mathrm{n}$ & $\%$ & $\mathrm{n}$ & $\%$ \\
\hline IBS-C & 6 & 15 & 5 & 20.83 & 11 & 17.18 \\
\hline IBS-D & 1 & 2.5 & 2 & 8.33 & 3 & 4.69 \\
\hline IBS-M & 1 & 2.5 & 3 & 12.5 & 4 & 6.25 \\
\hline IBS-U & 3 & 7.5 & - & - & 3 & 4.69 \\
\hline Total & 11 & 27.5 & 10 & 41.66 & 21 & 32.81 \\
\hline
\end{tabular}

Table 7. Mean IL-6 levels ((pg/ml)) in the studied groups

\begin{tabular}{|c|c|c|c|c|c|c|c|c|}
\hline \multicolumn{3}{|c|}{ IBS group $(n=40)$} & \multicolumn{3}{c|}{ SLE/IBS $(n=24)$} & \multicolumn{3}{c|}{ Control $(n=20)$} \\
\hline $\begin{array}{c}\text { PCR +ve } \\
(n=11)\end{array}$ & $\begin{array}{c}P C R-v e \\
(n=39)\end{array}$ & $P$ & $\begin{array}{c}P C R+v e \\
(n=10)\end{array}$ & $\begin{array}{c}P C R-v e \\
(n=14)\end{array}$ & $P$ & $\begin{array}{c}P C R+v e \\
(n=4)\end{array}$ & $\begin{array}{c}P C R-v e \\
(n=16)\end{array}$ & $P$ \\
\hline $15.9 \pm 3.5$ & $7.3 \pm 1.0$ & 0.0001 & $29.2 \pm 2.9$ & $10.1 \pm 1.5$ & 0.0001 & $13.8 \pm 1.8$ & $5.4 \pm 0.7$ & 0.003 \\
\hline
\end{tabular}

Blastocystis sp. from IBS patients in the present study suggests the possible causal role for IBS. Among 40 IBS patients positive for Blastocystis sp, 15\% (6/40) belonged to IBS-C followed by $7.5 \%$ (3/40) IBS-U and $2.5 \%$ (1/40) for both IBS-D and $M$ type. Using conventional PCR assay, Yakoob et al, (26), had reported a higher prevalence of Blastocystis sp. in an IBS-D clinical subtypes in Pakistan ( $44 \%$ vs. $21 \%$ in controls, $\mathrm{p}<0.001)$. On the contrary, study by
Nourrisson et al,(27) from France, did not show any prevalence. The disparity in prevalence of Blastocystis sp.in each clinical subtype of IBS may possibly be explained by the number of IBS patients enrolled in the respective studies. Blastocystis sp. subtype ST 3 was the most common subtype in the present study. In IBS group, it represented $17.5 \%$ (7/40), in SLE/IBS group, it represented $25 \%$ (6/24) and finally in control group, it represented half of positive 
samples (2/4). According to the recent study from India(28), ST3 subtype was found in all 27 normal healthy individuals positive for Blastocystis sp. and only in 2 samples showed mixed infection of both ST1 and ST3.

A. Blastocystis hominis $P C R$ patterns in IBS group
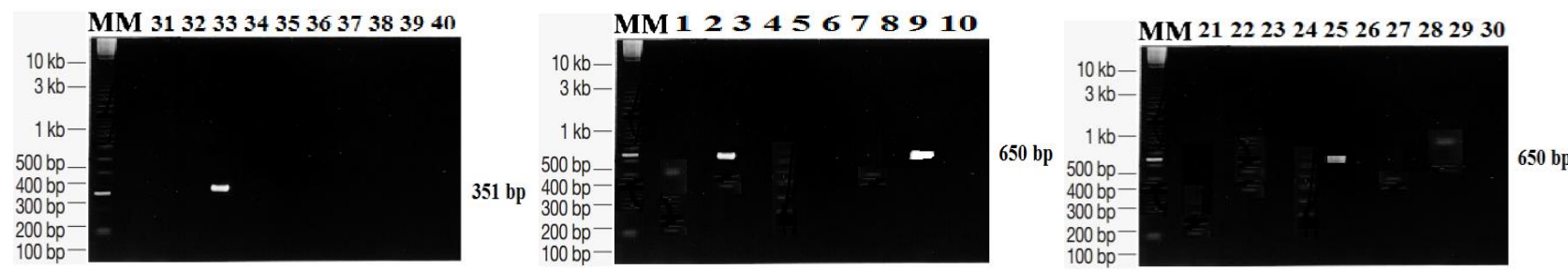

Subtype 1 SB83 IBS group Subtype 2 SB155 IBS group
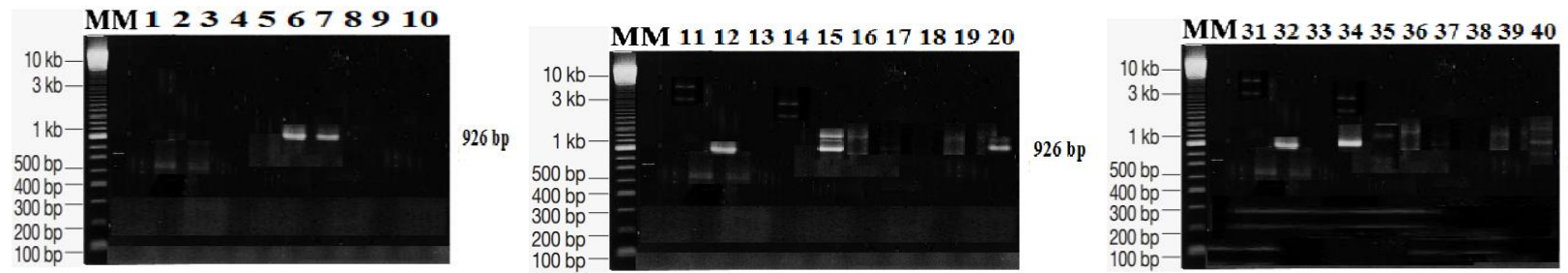

Subtype 3 SB227 IBS group

B. Blastocystis hominis PCR patterns in IBS - SLE group
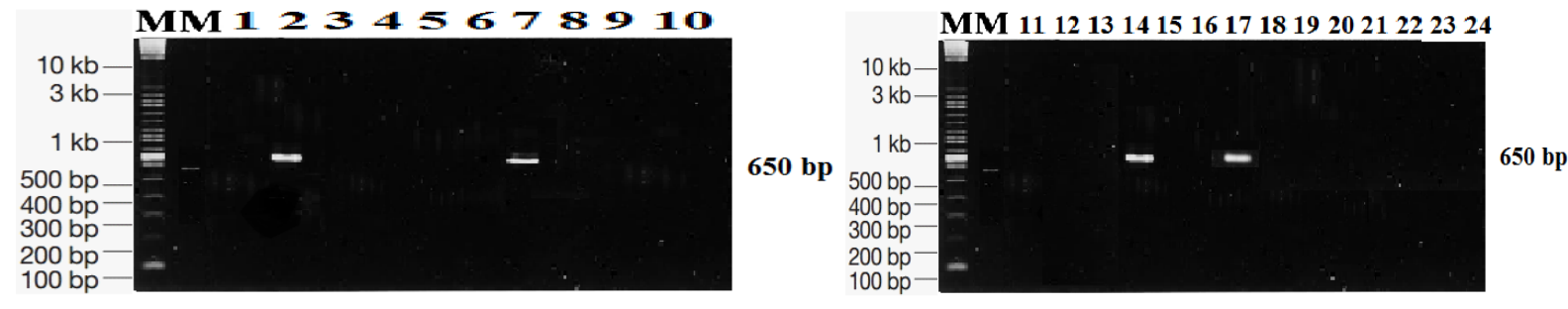

Subtype 2 SB155 IBS-SLE group
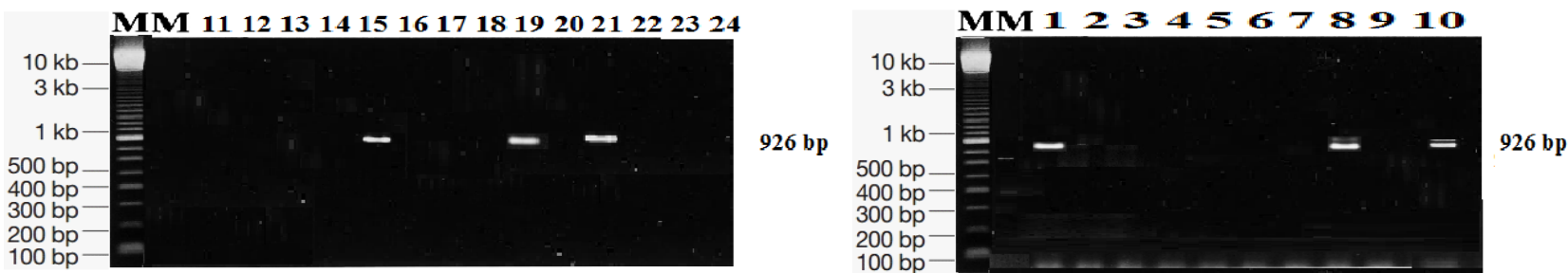

Subtype 3 SB1227 IBS-SLE group

C. Blastocystis hominis PCR patterns in control group
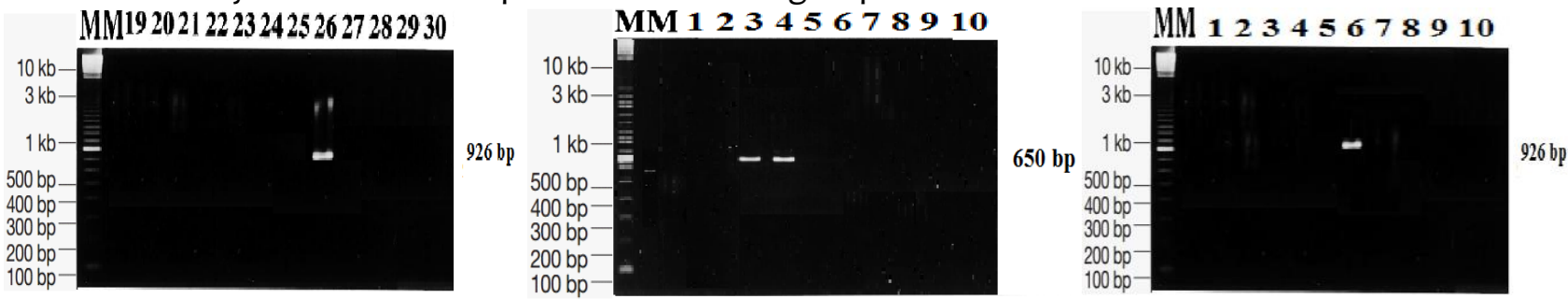

Subtype 2 SB155 control group Subtype 3 SB227 control group

Figure 1: Stained agarose gel with PCR products showing diagnostic bands at $351 \mathrm{bp}$ in ST1, 650 bp in ST2 and $926 \mathrm{bp}$ in ST3. $M$ is the marker at $100 \mathrm{bp}$. at different study groups 
In one of the comprehensive study by Yoshikawa et al, ${ }^{(29)}$, ST3 was the dominant subtype in the four different populations from different countries that included Japan, Bangladesh, Pakistan, and Germany, with a frequency ranging from $41.7 \%$ to 92.3\%.Study from Columbia has also shown marked association of ST1 being commonly isolated from asymptomatic individuals, ST2 from patients presenting with diarrhea and ST3 exclusively in patients with IBS(30). The difference in the relative diversity and prevalence of subtypes of Blastocystis sp. probably reflects epidemiological and demographic differences including climatic conditions, geographical attributes, cultural habits, proximity and exposure to reservoir hosts, and mode of transmission(31). A study of colonic biopsies of IBD patients showed that GIT symptoms is related to serine protease production ${ }^{(32)}$ which is produced by protozoa to antagonize host immunological factors, such as $\lg A^{(33)}$; such anti-Blastocystis IgA was involved in all symptomatic, but not asymptomatic, cases of Blastocystis(34). From this stand point; the theory of that the host attacks the protozoan not the reverse and a resultant disease manifestation is due to the protozoan's defence may explain why carriers of the parasite do not manifest symptoms (35). Many Parasites are incriminated in diseases of immune nature like arthritis; either by direct joint invasion or by toxin induced production of immune complexes, which leads to exaggerated immune response giving a reason to consider parasitic infestation in cases of autoimmune arthritis like Toxocara canis( ${ }^{(36)}$, Brachiola algerae $^{(37)}$, Cryptosporidum spp(38), Giardia lamblia(39), Strongyloides stercoralis(40). Furthermore; in Jamaica, physicians report of a female patients with severe arthritis affecting her knees poorly responded to steroid therapy and examination of synovial fluids revealed Blastocystis hominus indicating that it had migrated from the gut to the knee and arthritic activity relieved after metronidazol use ${ }^{(41)}$. Many reports exists about the relation between Blastocystis hominis and disease conditions like chronic urticaria and Hashimoto's thyroiditis which are well known autoimmune process and the suggested mechanisms was thought to be due to immune activation induced by the parasite through upregulation to variety of cytokines like IL-1 $\beta$, IL-6, and TNF- $\alpha^{(42)}$. SLE is an autoimmune disease of uncertain pathogenesis, characterized by deposition of autoantibodies and immune complexes in various organs leading to systemic manifestations including anywhere in the gut and may be termed lupus enteritis ${ }^{(43)}$ but most of SLE diagnostic criteria do not include them. However oral ulcers are the most common presentation encountered in up to $50 \%$ patients; while small and large intestinal abnormalities in SLE may demonstrate itself as dysmotility, vasculitis and malabsorption ${ }^{(44)}$. Protein-losing enteropathy may be a rare presentation of SLE, and its pathological background and management are not well understood (45) and the association of SLE with IBD is extremely rare ${ }^{(46)}$. One of the cytokines that modulates SLE activity and has a key action not just in its autoimmune pathogenesis is IL-6 but also ${ }^{(47)}$ in local inflammation like lupus nephritis(48), lupus carditis, lupus pneumonitis(49), and arthritis(50) and even in neuropsychiatric manifestations; high IL- 6 levels can be detected in cerebrospinal fluid( ${ }^{(51)}$; this may justify the rationale of this study to investigate IL- 6 in GIT manifestation in SLE. Taking in consideration that Blastocystis hominis can cause a leaky gut, which may be a manifestation in many, if not all autoimmune conditions as stated earlier together with it evident association to many cytokines ; the present study offered an evidence for a possible link of 
Blastocystis hominus and SLE disease process as $41.66 \%$ of the studied SLE patients has Blastocystis hominis infestation proved by PCR mainly subtypes 2 and 3 which is significantly higher when compared to patients with IBS or healthy controls. Moreover; IL-6 assay was higher in PCR positive patients in all study groups confirming the IL-6 / Blastocystis hominus pathogenic link and it is noted that the highest mean IL-6 level was in SLE patients which confirms the study hypothesis. At present; there is insufficient data about the immunomodulatory effect of Blastocystis infestation and the occurrence of SLE, but this study might show an association between both as shown by the significant rise of IL- 6 in SLE patients suffering of IBS hope for more researches in this area.

\section{Conclusion}

The prevalence of Blastocystis hominis infestation is higher in SLE patients who had IBS compared to IBS patients or asymptomatic controls; with evidence of IL- 6 increase in their sera suggesting an immunomodulatory interaction between SLE and Blastocystis hominis.

\section{References}

1. Kang JY. "Systematic review: the influence of geography and ethnicity in irritable bowel syndrome," Aliment Pharmacol Ther, vol. 21, no. 6, pp. 663-676, 2005.

2. Douglas R. Morgan, Matthew Benshoff, et al 2012: Irritable Bowel Syndrome and Gastrointestinal Parasite Infection in a Developing Nation Environment Hindawi Publishing Corporation Gastroenterol Res Pract Volume 2012, Article ID 343812, doi:10.1155/2012/343812

3. Nanthiney Devi Ragavan1, Suresh Kumar1, Tan Tian Chye1, Sanjiv Mahadeva2, Ho Shiaw- Hooi2. Blastocystis sp. in Irritable Bowel Syndrome (IBS) - Detection in Stool Aspirates during Colonoscopy.
PLOS ONE | DOI:10.1371/journal.pone .0121173 September 16, 2015

4. Wensaas A, Langeland N, Hanevik K, Mørch K, Eide GE, and Rortveit G. Irritable bowel syndrome and chronic fatigue 3 years after acute giardiasis: historic cohort study. Gut, vol. 61, no. 2, pp. 214219, 2012.

5. Tan TC, Suresh KG. Evidence of plasmotomy in B. hominis. Parasitol Res. 2007; 101: 1521-1525

6. Alfellani M, Stensvold C, Vidal-Lapiedra A, Onuoha E, Fagbenro-Beyioku A, Clark C. (2013) Variable geographic distribution of Blastocystis subtypes and its potential implications. Acta Trop 126: 11-18

7. Chandramathi S, Suresh K, Anita ZB, Kuppusamy UR. Infections of Blastocystis hominis and Microsporidia in cancer patients: are they opportunistic? Trans R Soc Trop Med Hyg. 2012; 106: 267-9.

8. Long $\mathrm{H}$, Handschack $\mathrm{A}$, Konig $\mathrm{W}$, Ambrosch A. (2001) Blastocystis hominis modulates immune responses and cytokine release in colonic epithelial cells. Parasitol Res 87: 1029-1030

9. Puthia M, Lu J, Tan K. (2008) Blastocystis ratti contains cysteine proteases that mediate interleukin- 8 response from human intestinal epithelial cells in an NF-kappaB-dependent manner. Eukaryot Cell 7: 435-443.

10. Alfellani MA, Taner-Mulla D, Jacob AS, et al. 2013. Genetic diversity of Blastocystis in livestock and zoo animals. Protist 164:497-509.

11. Cojocaru M, Cojocaru Inimioara Mihaela, SILOSI Isabela and VRABIE Camelia Doina, Gastrointestinal Manifestations in Systemic Auto-immune Diseases. Maedica (Buchar). $2011 \mathrm{Jan}$; 6(1): 45-51.

12. Grondal G, Gunnarsson I, Ronnelid J, Rogberg S, Klareskog L, and Lundberg I, "Cytokine production, serum levels and disease activity in systemic lupus erythematosus," Clin Exp Rheumatol, vol. 18, no. 5, pp. 565-570, 2000.

13. Tackey E, Lipsky P. E, and Illei GG, "Rationale for interleukin-6 blockade in 
systemic lupus erythematosus," Lupus, vol. 13, no. 5, pp. 339-343, 2004.

14. Abdel Galil SM, Ezzeldin N, El-Boshy ME. The role of serum IL-17 and IL- 6 as biomarkers of disease activity and predictors of remission in patients with lupus nephritis. Cytokine. 2015.

15. Yu C, Gershwin ME, Chang C. Diagnostic criteria for systemic lupus erythematosus: a critical review. J Autoimmun. 2014 Feb-Mar;48-49:10-3.

16. Yoshikawa H, Abe N, Iwasawa M, Kitano S, Nagano I, Wu Z, Takahashi Y 2000. Genomic analysis of Blastocystis hominis strains isolated from two long-term health care facilities. J Clin Microbiol 38: 1324-1330.

17. Stensvold CR, Suresh GK, Tan KSW, et al. 2007. Terminology for Blastocystis subtypes - a consensus. Trends Parasitol 23: $93-96$

18. Drossman D and Hasler W. Rome IVFunctional GI Disorders: Disorders of Gut-Brain Interaction Gastroenterology Vol. 150 , No. 6

19. Boorom KF, Smith H, Nimri L, Viscogliosi E, Spanakos G, Parkar Uetal. 2008. Oh my aching gut: irritable bowel syndrome, Blastocystis, and asymptomatic infection. Parasite Vectors 1(1):40.

20. Poirier P, Wawrzniak I, Vivares CP, Delbac F, ElAlaoui H. 2012. New insights into Blastocystis sp.: A potential link with Irritable Bowel Syndrome. Plos Path 8(3):e1002545

21. Stensvold R, Brillowska Dabrowska A, Nielsen HV, and Arendrup MC. (2006). Detection of Blastocystis hominis in unpreserved stool specimens by using polymerase chain reaction. J Parasitol 92, 1081-7.PMID:17152954

22. Dogruman-AIF, Simsek Z, Boorom K, Ekici E, Sahin M, Tuncer $C$ et al. 2010. Comparison of methods for detection of Blastocystis infection in routinely submitted stool samples, and also in IBS/IBD Patients in Ankara, Turkey. PLoS One (11): e15484.

23. Giacometti A, Cirioni O, Fiorentini A, Fortuna M, Scalise G. 1991. Irritable bowel syndrome in patients with
Blastocystis hominis infection. Eur J Clin Microbiol Infect Dis 18(6):436-9.

24. Surangsrirat $S$, Thamrongwittawatpong L, Piyaniran W, Naaglor T, Khoprasert C, Taamasri $P$ et al. 2010. Assessment of the association between Blastocystis infection and irritable bowel syndrome. J Med Assoc Thai 93(6):S119-24.

25. Ramirez-Miranda ME, Hernandez-Castellanos R, Lopez-Escamilla E, Moncada D, Rodriguez-Magallan A, PagasaMelero C. et al. (2010) Parasites in Mexican patients with irritable bowel syndrome: a case-control study. Parasit Vectors 3:96.

26. Yakoob J, Jafri W, Beg MA, Abbas Z, Naz $S$ et al. 2010. Irritable bowel syndrome: is it associated with genotypes of Blastocystis hominis. Parasitol Res 106(5):1033-8.

27. Nourrisson C, Scanzi J, Pereira B, Nkoud Mongo C, Wawrzyniak I, Cian A. et al. 2014. Blastocystis Is Associated with Decrease of Fecal Microbiota Protective Bacteria: Comparative Analysis between Patients with Irritable Bowel Syndrome and Control Subjects. PLoS One 9 (11): e111868.

28. Pandey PK, Verma P, Marathe N, Shetty S, Bavdekar A, Patole MA et al. 2015. Prevalence and subtype analysis of Blastocystis in healthy Indian Individuals. Infect Genetics Evol 31:296-299.

29. Yoshikawa H, Dogruman-AF, Turk S, Kustimur S, Balaban N, Sultan N. 2011. Evaluation of DNA extraction kits for molecular diagnosis of human Blastocystis subtypes from fecal samples. Parasitol Res 109(4):1045-50.

30. Ramírez JD, Sánchez LV, Bautista DC, Corredor AF, Flórez AC, Stensvold CR. 2014. Blastocystis subtypes detected in humans and animals from Colombia. Infect Genetics Evol 22:223-8.

31. Stensvold CR. 2013. Blastocystis: Genetic diversity and molecular methods for diagnosis and epidemiology. Trop Parasitol 3 (1): 2634AbeN. 2004. Molecular and phylogenetic analysis of Blastocystis isolates from various hosts. Vet Parasitol 120:235-242. 
32. Cenac N, Andrews CN, Holzhausen M, Chapman K, Cottrell G, Andrade- Gordon P, Steinhoff M, Barbara G, Beck P, Bunnet NW, Sharkey KA, Ferraz JG, Shaffer E, Vergnolle N 2007. Role for protease activity in visceral pain in irritable bowel syndrome. J Clin Invest 117: 636-647.

33. Puthia MK, Vaithilingam A, Lu J, Tan KS. Degradation of human secretory immunogloulin A by Blastocystis 2005. Parasitol Res 97: 386-389.

34. Mahmoud MS, Saleh WA 2003. Secretory and humoral antibody responses to Blastocystis hominis in symptomatic and asymptomatic human infections. J Egypt Soc Parasitol 33: 13-30.

35. Dogruman-F, Kustimur S, Yoshikawa H, et al. Blastocystis subtypes in irritable bowel syndrome and inflammatory bowel disease in Ankara, Turkey. Mem Inst Oswaldo Cruz, Rio de Janeiro, Vol. 104(5): 724-727, August 2009

36. Auer H, Aspock H. Nosology and epidemiology of human toxocarosis - the recent situation in Austria, Wiener Klinische Wochenschrift 116 Suppl 4:718, 2004

37. Cali A et al. An analysis of the microsporidian genus Brachiola, with comparisons of human and insect isolates of Brachiola algerae, J Eukaryot Microbiol NovDec 51(6):678-85, 2004

38. Sherry RQ. et al. Reiter's Syndrome associated with cryptosporidial gastroenteritis, J Rheumatol 22:1(Oct), 19621963, 1995.

39. Letts M. et al. Synovitis secondary to giardiasis in children, Am J Orthop 27:6 (June), 451-454, 1998.

40. Ghotekar LH. et al. Reactive arthritis, psoriasiform lesions and protein losing enteropathy secondary to Strongyloidiasis, J Assoc Physicians India Apr 51:3956, 2003.

41. Lee MG et al. Infective arthritis due to Blastocystis hominis, Ann Rheum Dis. Mar 49(3):192-3, 1990.

42. Xing Lim M, Wen Png C, Yan Bing Tay C, et al .Differential Regulation of Proinflammatory Cytokine Expression by
Mitogen-Activated Protein Kinases in Macrophages in Response to Intestinal Parasite Infection. Infect Immun. 2014 Nov; 82(11): 4789-4801.

43. Asherson R, Ramos-Casals M, Rodes J, et al. Digestive involvement in systemic autoimmune diseases. In Handbook of systemic autoimmune diseases Asherson R Elsevier Ltd. 2008;8:1-295.

44. Tian X-P, Zhang X. Gastrointestinal involvement in systemic lupus erythematosus: insight into pathogenesis, diagnosis and treatment. World J Gastroenterol. 2010;16:2971-7.

45. Zheng W, Tian X-P, Li L, et al. Protein-losing enteropathy in systemic lupus erythematosus: analysis of the clinical features of fifteen patients. J Clin Rheumatol. 2007; 13:313-6.

46. Lian TY, Edwards CJ, Chng HH. A study of a cohort of lupus patients with acute gastrointestinal manifestations of active disease. EULAR Conference (abstr); June 2001.

47. Linker-Israeli M, Deans RJ, Wallace DJ, Prehn J, Ozeri-Chen $\mathrm{T}$, and Klinenberg JR. Elevated levels of endogenous IL- 6 in systemic lupus erythematosus: a putative role in pathogenesis," J. Immunol, vol. 147, no. 1, pp. 117-123, 1991

48. Kishimoto T. Interleukin-6: from basic science to medicine: 40 years in immunology," Annu Rev Immunol., vol. 23, pp. 1-21, 2005

49. Yoshio T, Masuyama Jl, Kohda N. et al. Association of interleukin 6 release from endothelial cells and pulmonary hypertension in SLE," J Rheumatol., vol. 24, no. 3, pp. 489-495, 1997.

50. Eilertsen GO, Nikolaisen C, BeckerMerok A, and Nossent JC. Interleukin- 6 promotes arthritis and joint deformation in patients with systemic lupus erythematosus," Lupus, vol. 20, no. 6 , pp. 607-613, 2011.

51. Alcocer-Varela J, Aleman-Hoey D, and Alarcon-Segovia D. Interleukin-1 and interleukin- 6 activities are increased in the cerebrospinal fluid of patients with CNS lupus erythematosus and correlate 
with local late T-cell activation markers,

Lupus, vol. 1, no. 2, pp. 111-117, 1992. 